Nota Técnica

\title{
Registros documentados del Corocoro Escarlata (Eudocimus ruber Linnaeus, 1758; Aves: Threskiornithidae) en Loreto, Perú
}

[Documented records of Scarlet Ibis (Eudocimus ruber Linnaeus, 1758; Aves: Threskiornithidae) in Loreto, Peru]

Surecht Alberto Ruiz-Ramos ${ }^{* 1,2}$, Natalia Carolina Angulo-Perez ${ }^{3}$, Percy Saboya del Castillo4, Jorge Luis Gaviria Celis ${ }^{5}$, Juan Díaz Alván ${ }^{4}$

1. Asociación Civil Naturaleza Amazónica (NAAM), Jirón Cesar Calvo de Araujo Nº 914, Iquitos, Maynas, Loreto, Perú. Correo electrónico: surechtrr@gmail.com (S. A. Ruiz-Ramos* Autor de correspondencia).

2. Universidad Nacional de la Amazonía Peruana (UNAP). Facultad de Ciencias Biológicas. Pevas 5ta cuadra, Iquitos, Maynas, Loreto, Perú.

3. Instituto de Investigaciones de la Amazonía Peruana (IIAP). Av. A. Quiñones km 2,5, San Juan Bautista, Maynas, Loreto, Perú. Correo electrónico: nataliaangulo2797@gmail.com (N. C. Angulo-Perez).

4. Universidad Científica del Perú (UCP). Av. A. Quiñones km 2,5 (Iquitos), San Juan Bautista, Maynas, Loreto, Perú. Correos electrónicos: percnostola@gmail.com (P. Saboya), jdiaz@ucp.edu.pe (J. Díaz).

5. Servicio Nacional de Áreas Naturales Protegidas por el Estado (SERNANP). Parque Nacional Yaguas.

Correo electrónico: jgaviria991@gmail.com (J. L. Gaviria).

\section{Resumen}

El Corocoro Escarlata (Eudocimus ruber) está considerado para el Perú como una especie divagante, errante o ambas; su avistamiento ocurre ocasionalmente y no es parte de la avifauna habitual. En esta nota documentamos la presencia de Eudocimus ruber en Loreto, y se actualiza su ocurrencia en el Perú. Adicionalmente confirmamos que algunas especies de aves aparecen en la zona aledaña a la ciudad de Iquitos u otras áreas de Loreto como consecuencia de los nuevos espacios abiertos que son generados por la actividad antropogénica.

Palabras clave: Actividad antrópica, Divagante, Errante, Ocurrencia en el Perú.

\begin{abstract}
In Peru, the Scarlet Ibis (Eudocimus ruber) is considered a vagrant or errant species. Only recently has it been documented for Peru and its occurrence is occasional; not that of the habitual local avian community. In this note we present the registered accounts of this species in Loreto as well as outline its current status in Peru. Additionally, we confirm that some species of birds appear in the area surrounding the city of Iquitos or other areas of Loreto as a consequence of the new open spaces that are generated by anthropogenic activity.
\end{abstract}

Keywords: Anthropogenic activity, Errant, Occurrence in Peru, Vagrants. 


\section{INTRODUCCIÓN}

El Corocoro Escarlata (Eudocimus ruber), es una llamativa ave que se distribuye en Sudamérica, en los llanos de Colombia y Venezuela, Guayanas, Surinam, Trinidad y Tobago, en la zona este de Brasil a lo largo de la costa atlántica, desde Río de Janeiro hasta Santa Catarina; también reportado para Panamá (Hilty y Brown, 1986; Matheu, 2020) y en Ecuador donde es considerado como errante y muy raro (Ridgely y Greenfield, 2001; Nilsson et al., 2014). La especie se encuentra catalogada en Preocupación Menor (LC) (IUCN, 2020), aunque se sospecha que su población estaría disminuyendo por la continua destrucción de su hábitat (Birdlife International, 2020). Usualmente es observado hasta los $500 \mathrm{msnm}$, con preferencia de determinados hábitats como manglares, estuarios fangosos y marismas de marea, pantanos de agua dulce, lagos someros, humedales, arrozales, borde de río y estanques de aguas residuales y de peces (Hilty y Brown, 1986; Ridgely y Greenfield, 2001; Matheu, 2020).

Actualmente en Perú E. ruber fue agregado recientemente a su lista de aves (Angulo et al., 2019), por las primeros observaciones y registros documentados de la especie para el país llevadas a cabo el 12 de setiembre del 2017, por Ottavio Janni, Andrea Corso, Flor Peña y Michele Vigano en la cuenca alta del río Putumayo, zona fronteriza con Colombia, a $9 \mathrm{~km}$ aguas arriba de la localidad de Puerto Leguízamo (Colombia), en el lado peruano. Anteriormente no existía ninguna observación de la especie (Schulenberg et al., 2010). Esta nota científica tiene como objetivo enriquecer el conocimiento de la presencia de E. ruber en Loreto, además de actualizar su ocurrencia en Perú.

\section{REGISTROS}

El 13 de abril del 2019, N.C. Angulo-Perez, observó, fotografió e hizo un vídeo de un individuo juvenil de Eudocimus sp. a las 10 horas, en los alrededores de la comunidad Remanso (Tabla 1, Figura 1), Distrito Yaguas, Provincia Putu- mayo, Departamento Loreto, cabe mencionar que en el estadío juvenil Eudocimus ruber y $E$. albus son muy parecidos, por tal motivo no podemos determinar con exactitud la especie, ya que, existen reportes de presencia de $E$. albus adultos en la cuenca alta del Putumayo, observadas por J. B. Socolar en setiembre del 2019 (https://ebird.org/checklist/S59799168). El individuo juvenil observado se encontraba aparentemente buscando alimento en un gran pasto de la comunidad (espacio abierto y cubierto principalmente por hierbas). Posteriormente se le observó desplazándose hacia una pequeña quebrada aledaño a la comunidad, acompañado por algunos individuos de la Garcita Bueyera (Bubulcus ibis), Gallinazo de cabeza negra (Coragyps atratus) y algunas aves de corral.

P. Saboya el 30 de abril del 2019 a las 15 horas observó y fotografió a dos individuos juveniles de Eudocimus sp. descansando y acicalándose en un cerco de madera de la huerta de una casa de la comunidad Remanso (Figura 2), lugar adyacente a los pastizales mencionados anteriormente. El 02 de mayo de 2019 a las 13 horas P. Saboya observó y fotografió nuevamente a los dos individuos juveniles de Eudocimus sp. en un caño entre los pastizales de la comunidad (Figura 3), mientras buscaban alimento escarbando y removiendo la tierra lodosa con su pico curvado y largo, junto a un individuo de Espátula Rosada (Platalea ajaja), individuos de la Garcita Bueyera (Bubulcus ibis) y el Avefría Tero (Vanellus chilensis).

S. A. Ruiz-Ramos el 04 octubre del 2019, observó 3 individuos adultos de Eudocimus ruber alimentándose de peces de forraje en horas de la mañana en un estanque piscícola dentro del fundo "Tony" (Tabla 1), ubicado a la altura del km 34,200 de la carretera Iquitos-Nauta, a 600 $\mathrm{m}$ aprox. en su margen izquierdo, en el Distrito San Juan Bautista, Provincia Maynas, Departamento Loreto. Los individuos fueron observados esporádicamente los siguientes 8 meses; la detección de los individuos ocurría posterior al desaguado de los estanques piscícolas ubicados en esta zona. 
Tabla 1. Registros del Corocoro Escarlata (Eudocimus ruber) y Eudocimus sp. en Loreto, Perú.

\begin{tabular}{|c|c|c|c|c|c|c|c|c|}
\hline $\mathbf{N}^{\circ}$ & Observadores & $\begin{array}{l}\mathrm{N}^{\circ} \text { de } \\
\text { indivi- } \\
\text { duos }\end{array}$ & $\begin{array}{c}\text { Estadío de } \\
\text { madurez } \\
\text { sexual }\end{array}$ & Fechas & $\begin{array}{l}\text { Lugar de } \\
\text { observación }\end{array}$ & $\begin{array}{c}\text { Documen- } \\
\text { tado } \\
\text { (Fotografía) }\end{array}$ & Coordenadas & $\begin{array}{c}\text { Eleva- } \\
\text { ción } \\
\text { (msnm) }\end{array}$ \\
\hline 1 & Ottavio Janni* & 1 & Adulto & $31 / 01 / 2017$ & Río Putumayo & $\mathrm{Si}$ & $0^{\circ} 14^{\prime} 15,6^{\prime \prime} \mathrm{S} ; 74^{\circ} 51^{\prime} 34,6^{\prime \prime} \mathrm{O}$ & 180 \\
\hline 2 & Diego Calderón Franco* & 1 & - & $27 / 03 / 2017$ & Río Putumayo & - & $0^{\circ} 14^{\prime} 26,8^{\prime \prime} \mathrm{S} ; 74^{\circ} 52^{\prime} 37,3^{\prime \prime} \mathrm{O}$ & 180 \\
\hline 3 & Natalia Carolina Angulo Perez** & 2 & Juvenil & $13 / 04 / 2019$ & $\begin{array}{l}\text { Comunidad } \\
\text { Remanso }\end{array}$ & $\mathrm{Si}$ & $2^{\circ} 24^{\prime} 23,1^{\prime \prime} \mathrm{S} ; 71^{\circ} 10^{\prime} 38,5^{\prime \prime} \mathrm{O}$ & 88 \\
\hline 4 & Percy Saboya del Castillo** & 2 & Juvenil & $\begin{array}{l}30 / 04 / 2019 \\
02 / 05 / 2019\end{array}$ & $\begin{array}{c}\text { Comunidad } \\
\text { Remanso }\end{array}$ & $\mathrm{Si}$ & $2^{\circ} 24^{\prime} 23,1^{\prime \prime} \mathrm{S} ; 71^{\circ} 10^{\prime} 38,5^{\prime \prime} \mathrm{O}$ & 88 \\
\hline 5 & Surecht Alberto Ruiz Ramos & 3 & Adulto & 04/10/2019 & $\begin{array}{c}\text { Carretera } \\
\text { Iquitos-Nauta } \\
\text { (km 34) }\end{array}$ & - & $4^{\circ} 02^{\prime} 11,25^{\prime \prime} \mathrm{S} ; 73^{\circ} 25^{\prime} 51,31^{\prime \prime} \mathrm{O}$ & 103 \\
\hline 6 & Juan Díaz Alván & 2 & Adulto & $11 / 11 / 2019$ & $\begin{array}{l}\text { Comunidad } \\
\text { Tres Esquinas }\end{array}$ & - & $2^{\circ} 29^{\prime} 19,6^{\prime \prime} \mathrm{S} ; 70^{\circ} 35^{\prime} 50,47^{\prime \prime} \mathrm{O}$ & 80 \\
\hline 7 & Jorge Gaviria Celis & 1 & Juvenil & $15 / 12 / 2019$ & $\begin{array}{l}\text { Comunidad } \\
\text { Remanso }\end{array}$ & $\mathrm{Si}$ & $2^{\circ} 24^{\prime} 23,1^{\prime \prime} \mathrm{S} ; 71^{\circ} 10^{\prime} 38,5^{\prime \prime} \mathrm{O}$ & 88 \\
\hline 8 & Felix Solsol Ríos & 1 & Adulto & $28 / 05 / 2020$ & $\begin{array}{c}\text { Carretera } \\
\text { Iquitos-Nauta } \\
(\mathrm{km} \mathrm{34})\end{array}$ & $\mathrm{Si}$ & $4^{\circ} 02^{\prime} 11,25^{\prime \prime} \mathrm{S} ; 73^{\circ} 25^{\prime} 51,31^{\prime \prime} \mathrm{O}$ & 103 \\
\hline
\end{tabular}

* Datos obtenidos de la plataforma eBird (https://ebird.org/checklist/S59799168; https://ebird.org/checklist/S35725744)

** Juvenil de Eudocimus sp.

Fuente: Elaboración propia.

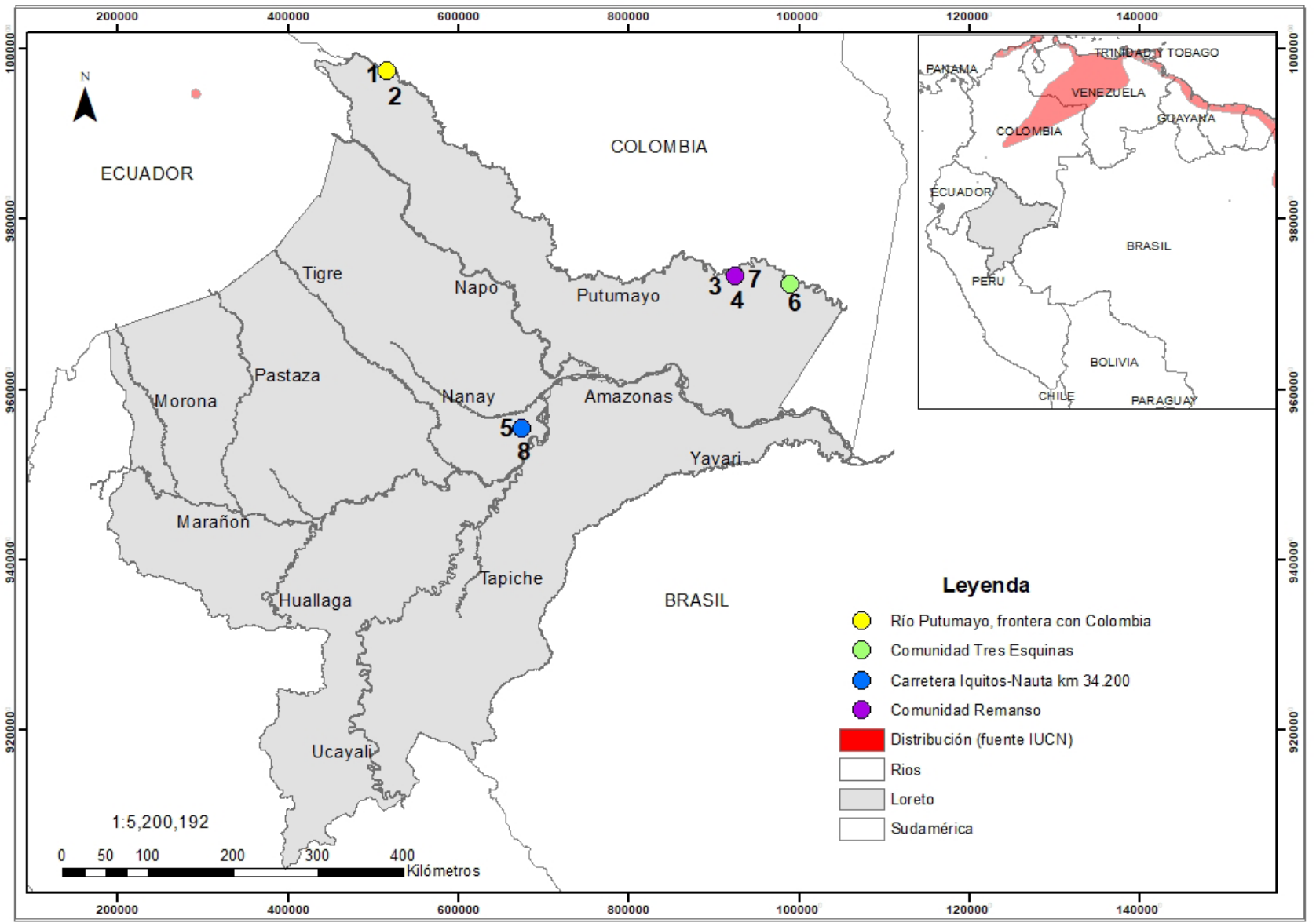

Figura 1. Mapa de registros del Corocoro Escarlata (Eudocimus ruber) y Eudocimus sp. en Loreto, Perú. 


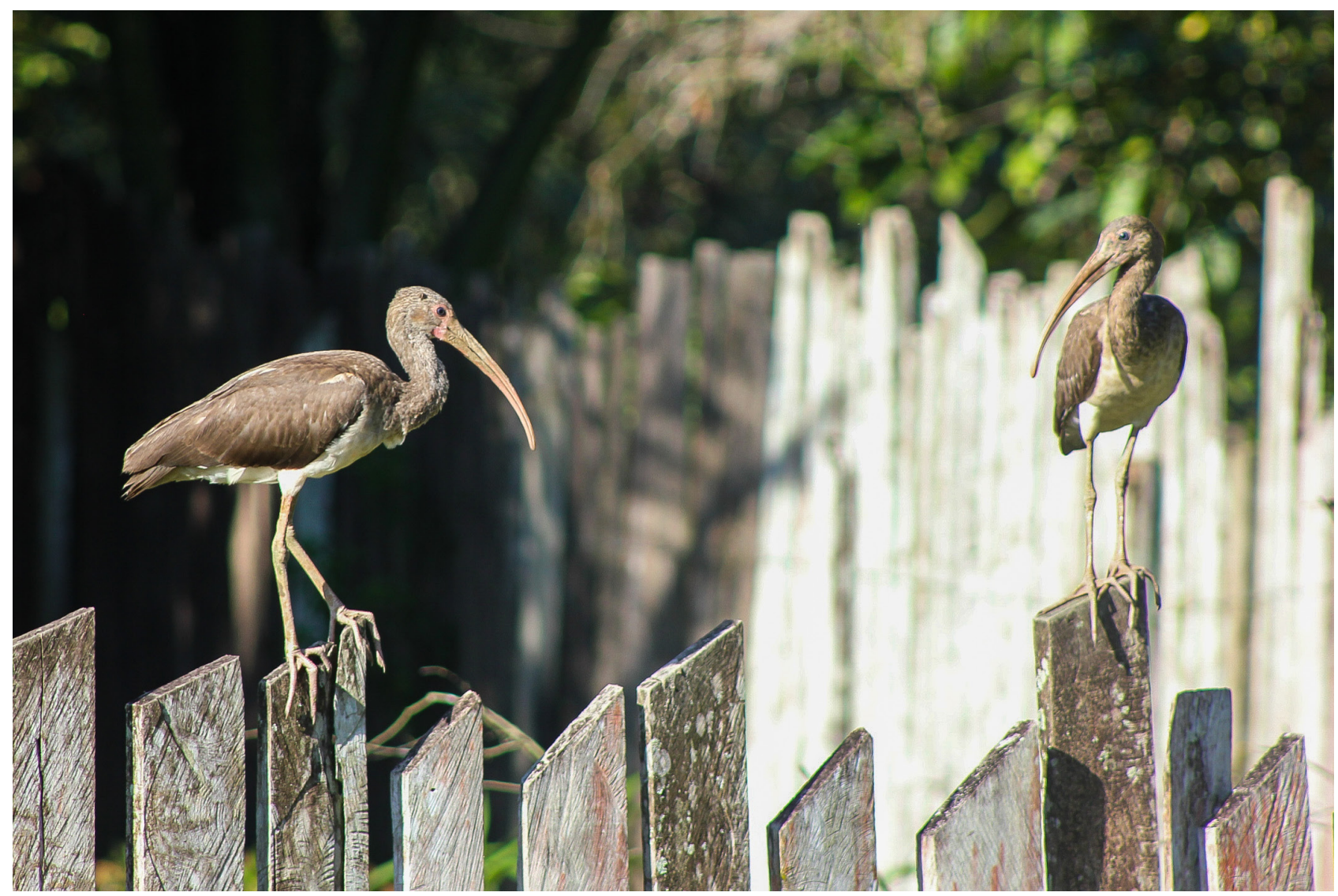

Figura 2. Individuos juveniles de Eudocimus sp. descansando en un cerco en la comunidad Remanso, río Putumayo. Abril del 2019. Foto: P. Saboya del Castillo.

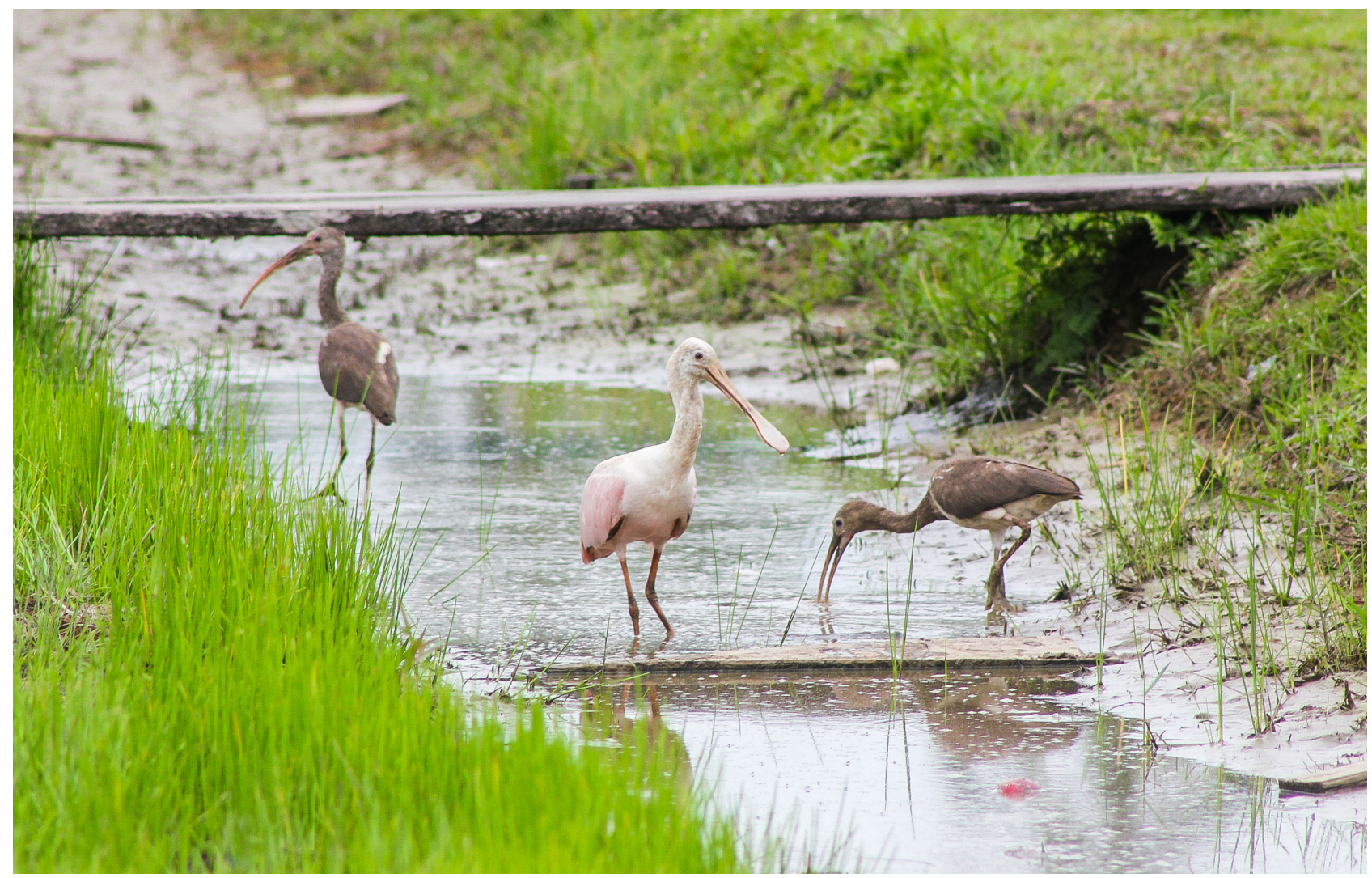

Figura 3. Individuos juveniles de Eudocimus sp. buscando su alimento en un caño, junto a un individuo de Platalea ajaja, comunidad Remanso, río Putumayo. Mayo del 2019. Foto: P. Saboya del Castillo. 
J. Díaz, el 10 de noviembre del 2019, observó a las 8 horas a un par de individuos adultos reposando en un árbol, próximos a la comunidad Tres Esquinas (Tabla 1, Figura 1), en el Distrito Yaguas, provincia Putumayo, en el departamento Loreto. Los individuos luego se desplazaron por el río Putumayo perdiéndose posteriormente en los bosques del lado colombiano del mencionado río.

J. L. Gaviria, el 15 de diciembre del 2019, observó y fotografío a las 16 horas y 30 minutos a un individuo de $E$. ruber en áreas cercanas a un pasto en la comunidad Remanso, en búsqueda de alimento (Figura 4), junto a varios individuos de Garza Blanca (E. thula). El individuo observado en esta ocasión presentaba plumaje juvenil y con un claro cambio de color hacia el plumaje adulto (coloración rojo o escarlata).

El 28 de mayo del 2020 a las 15 horas, se volvió a registrar a la especie en el Fundo Tony, en este caso fue un individuo adulto, el cual se encontraba alimentándose y posteriormente reposando en un árbol, fue fotografiado por Felix Ulises Solsol Ríos (Figura 5 y 6). En este lugar, los individuos ocasionalmente fueron observados buscando alimento en los estanques piscícolas junto a la Garza Grande (Ardea alba), Egretta thula, Gallito de Agua de Frente Roja (Jacana jacana) y la Espátula Rosada (Platalea ajaja). Las especies de peces de forraje que comúnmente se encuentran en estos estanques son: Fasaco (Hoplias malabaricus), Boquichico (Prochilodus nigricans), Bujurqui (Cichlasoma sp.) y Mojaras (Astyanax sp.), en algunas ocasiones estos peces ingresan accidentalmente de las quebradas que proporcionar agua a los estanques durante el proceso de recambio de agua, esta dieta coincide con lo reportado para la especie, aunque no se descarta que adicionalmente podría estar alimentándose de algunos crustáceos, tal como lo indica Matheu (2020).

En la Tabla 1 y Figura 1 se hace referencia a todos los registros llevadas a cabo por los autores y se adicionan registros de la plataforma eBird (https://ebird.org/home).

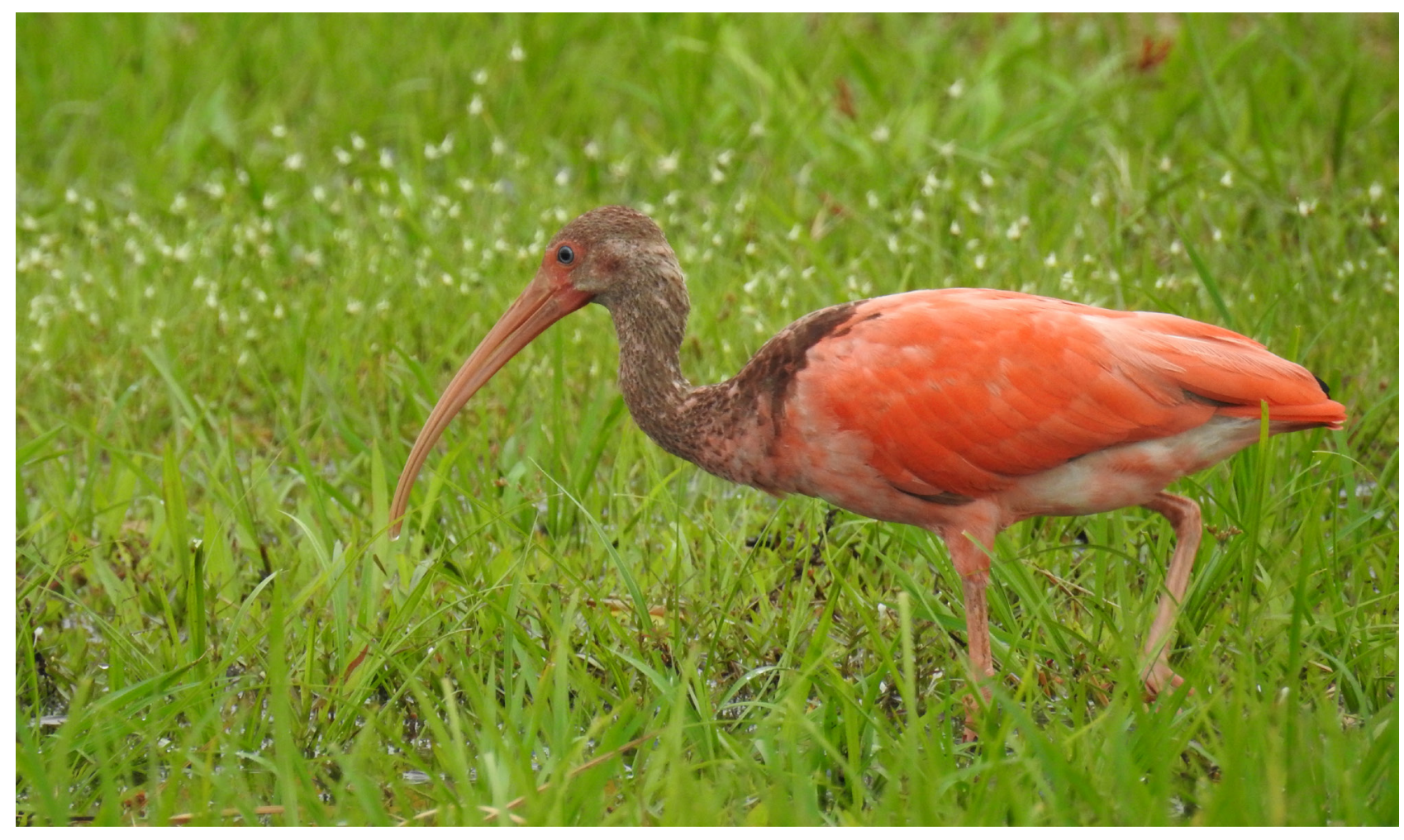

Figura 4. Individuo juvenil del Corocoro Escarlata ( $E$. ruber) buscando su alimento en un pasto, comunidad Remanso, río Putumayo. Diciembre del 2019. Foto: J. L. Gaviria Celis. 


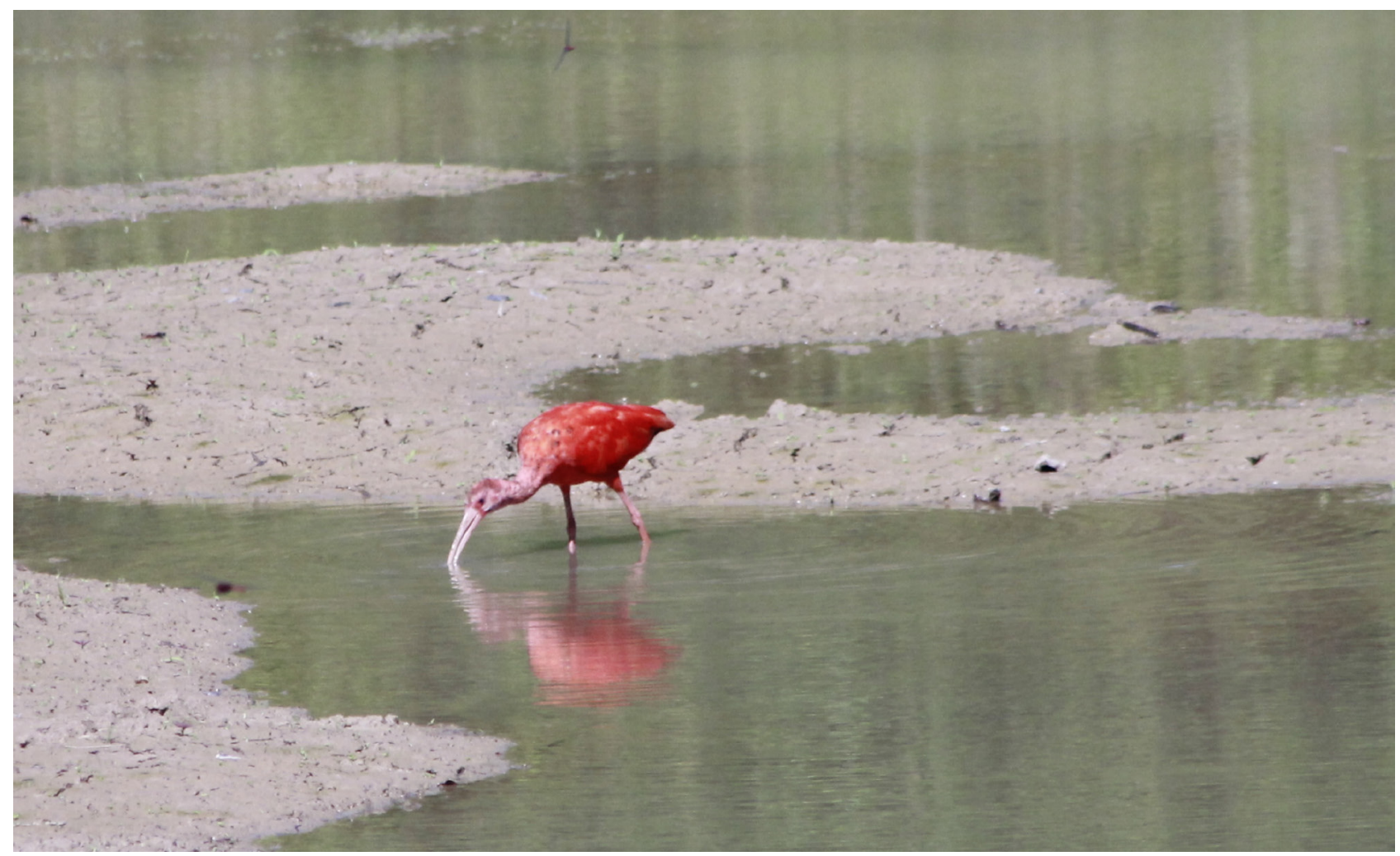

Figura 5. Individuo adulto del Corocoro Escarlata (E. ruber) buscando su alimento, en un estanque piscícola, dentro del Fundo Tony, Carretera Iquitos-Nauta. Mayo del 2020. Foto: Felix U. Solsol Ríos.

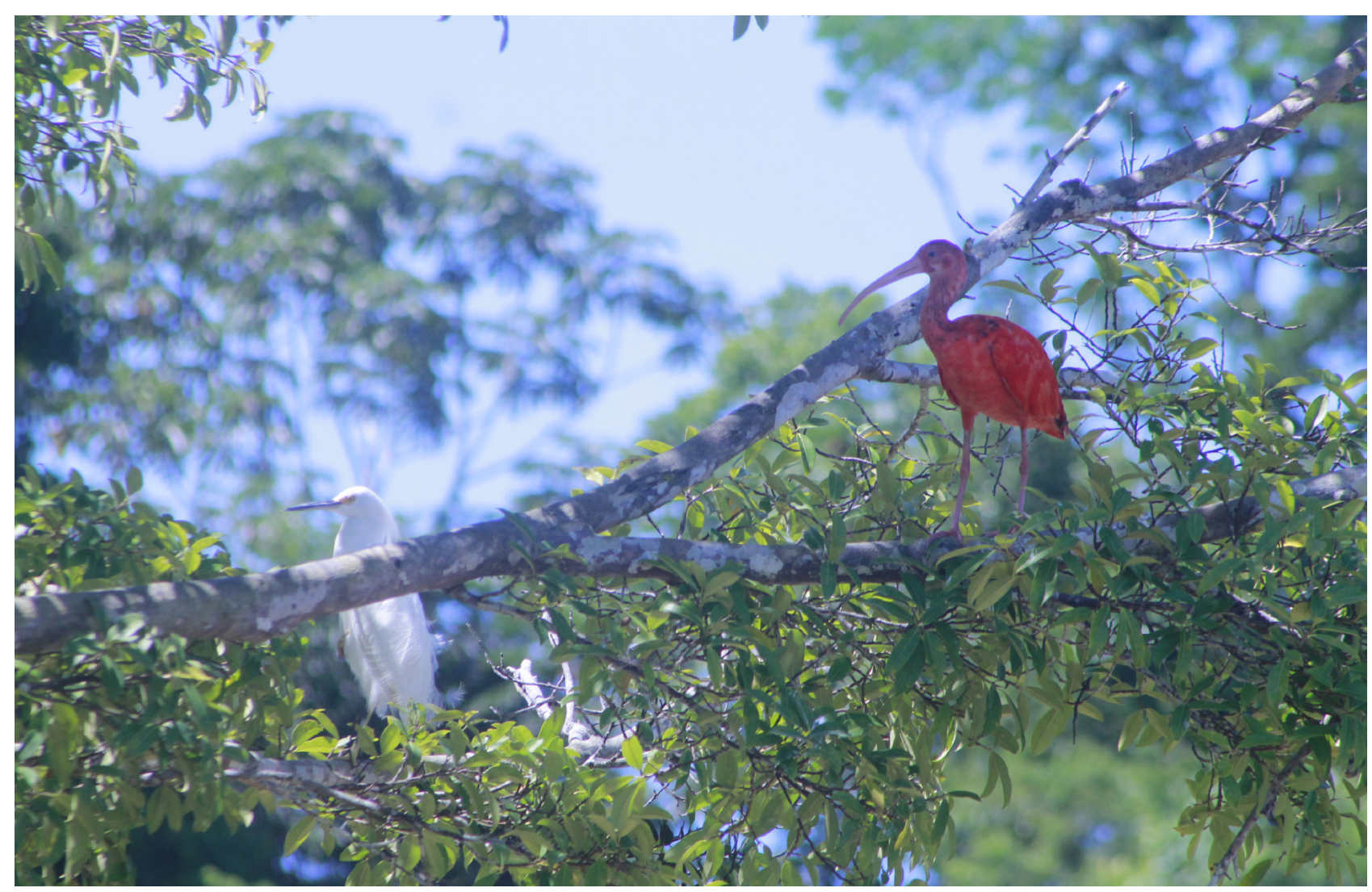

Figura 6. Individuo adulto del Corocoro Escarlata (E. ruber) reposando en un árbol, junto a un individuo de Egretta thula dentro del Fundo Tony, Carretera Iquitos-Nauta. Mayo del 2020. Foto: Felix U. Solsol Ríos. 


\section{DISCUSIÓN}

De acuerdo con nuestros registros de avistamiento y otros recientemente publicados (Angulo et al., 2019), E. ruber vendría ampliando su rango de distribución desde la cuenca del río Putumayo, $400 \mathrm{~km}$ desde la zona de Leguízamo y $300 \mathrm{~km}$ desde las comunidades de Remanso y Tres Esquinas, hacia la ciudad de Iquitos, en el Departamento de Loreto, entre los 80 y 180 msnm. La especie se encontraría aprovechando el incremento de hábitats apropiados para su establecimiento a lo largo de los grandes ríos como el Putumayo, posiblemente río Amazonas y en la carretera Iquitos-Nauta, especialmente por la actividad antropogénica es decir, áreas con bosque secundario, espacios abiertos cercanos a las comunidades, estanques piscícolas que se convierten en fuentes de alimento para la especie, tal como se observó en la carretera Iquitos-Nauta, lo que concuerda con lo reportado en la zona fronteriza entre Perú y Colombia (Bonilla-Castillo et al., 2017). Este mismo patrón se observó en otras especies de aves recientemente registradas en Loreto, que se benefician de estos nuevos espacios como resultado de actividades antropogénicas, entre ellas podemos citar al Caracara Carancho Caracara plancus (Piana et al., 2012), Avefría Tero Vanellus chilensis (Ruiz-Ramos et al., 2017), Zambullidor Menor Tachybaptus dominicus (Ruiz-Ramos et al., 2018), Ibis de Cara Pelada Phimosus infuscatus (Socolar et al., 2018), Calandria Tropical Mimus gilvus (Cuelo, 2018).

Los registros de los individuos adultos y juvenil de $E$. ruber, probablemente nos esté evidenciando su establecimiento en un futuro como una especie residente, aunque falta comprobar su presencia de manera constante a lo largo de todo el año. De acuerdo con los moradores de la comunidad Remanso, los registros de la especie en este lugar datan del 2017, lo cual nos indican que son observaciones consistentes y nos ayuda a entender que son sostenidos en el tiempo. Así mismo considerando su rápida expansión hacia la zona de Iquitos, no sorprendería que la especie se estuviera estableciendo en territorio peruano, tal como se observa en la zona fronteriza con Colombia, quienes probablemente se estarían desplazándose desde los llanos de Colombia o Venezuela, por ello podría dejar de ser calificada en algunos pocos años como una especie errante, tal como es considerada en la actualidad (Plenge, 2020; Wiley et al., 2018).

Finalmente consideramos que es importante el registro y reporte documentado de especies que van apareciendo en territorio peruano $y$ también aprovechar plataformas virtuales de registro de especies como eBird (https://ebird. org/home) para este fin y con información adecuada y detallada (número de individuos, estado de madurez, hábitat, lugar exacto de observación, etc.), que será de mucha utilidad para futuros estudios que puedan ayudar a monitorear el impacto de la actividad antropogénica en la Amazonía peruana.

\section{AGRADECIMIENTOS}

Agradecemos al señor Felix Ulises Solsol Ríos por brindarnos las facilidades para ingresar a sus instalaciones (Fundo Tony), a Alonso Armas por el apoyo en la fotografía. Adicionalmente agradecemos a Brayan Coral, Diego Calderón, Michele Viganò y Ottavio Janni por el reporte de sus observaciones en la página de eBird y a Juan Freile. A Fernando Angulo, Antonio García-Bravo, Jacob B. Socolar y un revisor anónimo por las sugerencias que mejoraron sustancialmente el documento. Agradecemos también a la IUCN por permitirnos usar el mapa de distribución de E. ruber.

\section{REFERENCIAS BIBLIOGRÁFICAS}

Angulo, F., Begazo, A., Lane, D., Plenge, M., Stotz, D., Ugarte, M., Valqui, V. y Walker, B. (2019) Reporte del Comité de Registros de Aves Peruanas (CRAP) del periodo 2017. Boletín de la Unión de Ornitólogos del Perú (UNOP), 14 (2), 51-62.

BirdLife International (2020) Species factsheet: Eudocimus ruber [en línea], Disponible en 
<http://datazone.birdlife.org/species/factsheet/scarlet-ibis-eudocimus-ruber>. [Consulta: 30 mayo 2020].

Bonilla-Castillo, C., Peña, F., Bonilla-Velazquez, C. y Velazquez-Figueroa, I. (2017) La corocora (Eudocimus ruber) en la llanura amazónica entre los ríos Caquetá y Putumayo. Ornitología Colombiana, 16, 1-1.

Cuelo, P. (2018) Primer registro documentado de Calandria Tropical (Mimus gilvus) para el Perú. Boletín de la Unión de Ornitólogos del Perú (UNOP), 13 (1), 7-9.

eBird. (2020) Una base de datos en línea para la abundancia y distribución de las aves. [en línea], Disponible en <https://ebird.org/ home> [Consulta: 20 abril 2020.

Hilty, S. y Brown, W. (1986) A guide to the birds of Colombia. Princeton, NJ: Princeton University Press.

Matheu, E., Del Hoyo, J., Garcia, E. y Boesman, P. (2020) Scarlet Ibis (Eudocimus ruber), version 1.0. In Birds of the World (Del Hoyo, J., Elliott, A., Sargatal, J., Christie, D. y De Juana, E., Editors). Cornell Lab of Ornithology, Ithaca, NY, USA. [en línea], Disponible en <https://doi.org/10.2173/bow.scaibi.01> [Consulta: 20 abril 2020].

Nilsson, J., Freile, J., Ahlman, R., Brinkhuizen, D., Greenfield, P. y Solano-Ugalde, A. (2014) Rare birds in Ecuador: second annual report of the Committee for Ecuadorian Records in Ornithology (CERO). Avances en Ciencias e Ingenierías, 6 (2), B38-B50.

Piana, R., Díaz, A., Chalco, L., Sevillano, C., Saboya, del C. y Cubas, P. (2012) El Caracara Carancho (Caracara plancus) estaría expandiendo su rango de distribución hacia el norte y oeste de la Amazonía peruana. Boletín de la Unión de Ornitólogos del Perú (UNOP), 7 (2), 5-11.

Plenge, M. (2020) Lista de aves del Perú. Boletín de la Unión de Ornitólogos del Perú. [en línea], Disponible en: <https://sites.google. com/site/boletinunop/checklist.> [Consulta: 20 abril 2020].

Ridgely, R. y Greenfield, P. (2001) The Birds of Ecuador, Volume 2. Ithaca, NY: Cornell University Press.
Ruiz-Ramos, S., Alegría-Torres, B., Ayapi-Da-Silva, J. y Díaz-Alván, J. (2017) Registro documentado del Avefría Tero (Vanellus chilensis) en selva Norte del Perú. Boletín de la Unión de Ornitólogos del Perú (UNOP), 12 (1), 15-18.

Ruiz-Ramos, S., Ayapi-Da-Silva, J., Alegría-Torres, B. y Díaz, A. (2018) Registro documentado del Zambullidor Menor (Tachybaptus dominicus) en la selva Norte de Loreto. Boletín de la Unión de Ornitólogos del Perú (UNOP), 13 (2), 12-15.

Schulenberg, T., Stotz, D., Lane, D., O'Neill, J., Parker III, T. (2010) Birds of Peru. Revised and updated edition. Princeton, NJ: Princeton University Press.

Socolar, J., Díaz, A., Saboya, Del C., Pomara, L., O'Shea, B., Cubas, P., Stotz, D., Schmitt, F., Graham, D., Carnes, B. y Ruelas, I. (2018) Noteworthy bird records from northeastern Peru reveal connectivity and isolation in the western Amazonian avifauna. The Wilson Journal of Ornithology, 130 (1), 94-111.

IUCN (2020) The IUCN Red List of Threatened species. 2020. Version 2020-1. [en línea], Disponible en: <https://www.iucnredlist. org.> [Consulta: 20 junio 2020].

Wiley, R., Álvarez, A. y Díaz A. (2018) Aves de Loreto bajo (60-250 m SNM/ASL). Topazapyra press, Lima, Perú.

\section{Conflicto de interés}

Los autores declaramos no tener ningún conflicto de interés. 